\title{
Differences in Breast Density Awareness, Knowledge, and Plans Based on State Legislation Status and Sociodemographic Characteristics
}

\author{
Nancy R. Kressin, $P h D^{1,2}$ (D) , Jolie B. Wormwood, $P h D^{3}$, Tracy A. Battaglia, MD, MPH ${ }^{2}$, and \\ Christine M. Gunn, PhD 2
}

'Veterans Affairs Boston Healthcare System, Boston, MA, USA; ${ }^{2}$ Section of General Internal Medicine, Department of Medicine, Boston University School of Medicine, Boston, MA, USA; ${ }^{3}$ Department of Psychology, University of New Hampshire, Durham, NH, USA.

J Gen Intern Med 35(6):1923-5

DOI: $10.1007 / \mathrm{s} 11606-019-05578-3$

(c) Society ofGeneral InternalMedicine (This is aU.S. government work and not under copyright protection in the U.S.; foreign copyright protection may apply 2019

\section{INTRODUCTION}

State-level legislation requires informing women about breast density (BD) with mammogram results, to increase awareness of BD's tendency to mask cancers on mammography, association with increased breast cancer risk, and encourage women to discuss personal risk and supplemental screening with physicians. The Food and Drug Administration is developing dense breast notification (DBN) language for use nationwide; ${ }^{1}$ information about effects of state DBNs could be informative.

\section{METHODS}

We assessed BD awareness, knowledge, reactions to DBNs, and past/planned discussions with physicians, adapting prior questions $^{2}$ for inclusion in a national, weekly, bilingual random digit dial telephone survey (land lines and cell phones) targeting a representative sample of the US population. Women in all states aged $\geq 40$ reporting receipt of a mammogram in $\leq 2$ years were eligible. Bivariate and multivariate analyses examined how outcomes varied by state DBN status (whether women reside in states mandating DBNs) and women's sociodemographic characteristics (age, income, education, race/ethnicity).

\section{RESULTS}

The survey was presented to 691 women deemed eligible; 578 $(83.5 \%)$ completed it. Sociodemographic characteristics did not vary by state DBN status (not shown). The proportion of

The manuscript has not been published elsewhere nor is it under review elsewhere

Received August 29, 2019

Revised September 26, 2019

Accepted November 22, 2019

Published online December 16, 2019 women who had heard of breast density was $76 \%$, with no differences by state DBN status. In bivariate (not shown) and multivariate results (Table 1), women with less education, lower incomes, and non-white race/ethnicity were less likely to have heard of BD. Among women who had heard of BD, bivariate analyses showed that the percentage of women endorsing that $\mathrm{BD}$ means how breasts feel when one touches them was less/better among white women, in DBN states, with higher incomes, and college degrees; state DBN status and race/ethnicity remained significant in multivariate analyses. We observed no bivariate differences in whether BD refers to what breasts look like on a mammogram; $\geq 80 \%$ responded correctly; higher income, older women said "yes" more often in multivariate analyses. When asked whether BD makes it more difficult for a mammogram to correctly detect cancer, white and Hispanic women and those with higher incomes, more education, and aged 65+ were less often correct in bivariate analyses; none remained significant in multivariate results. There were no bivariate state DBN status differences regarding women's knowledge of whether dense breasts increase risk of breast cancer; less than half of women recognized this risk. Women with some college were more likely to correctly respond in bivariate but not in multivariate analyses. Among women reporting receiving a DBN, there were few bivariate differences in feeling informed or confused about $\mathrm{BD}$; in multivariate results, Black women reported significantly more anxiety and confusion about $\mathrm{BD}$; higher income women reported less. There were no differences in having discussed BD with a doctor, but among those not yet having had such discussions, all results indicated that women residing in DBN states, with higher incomes and non-Hispanic ethnicity were less likely to plan such discussions.

\section{DISCUSSION}

This national survey found few differences in outcomes by state DBN status but numerous differences disadvantaging lower socioeconomic status and racial/ethnic minority women. Results identified greater anxiety and confusion about BD among Black women, also found by others. ${ }^{3,4}$ 
Table 1 Multivariate models. Women's breast density awareness, knowledge, reactions, and follow-up plans, by state DBN legislation status and women's sociodemographic characteristics

\begin{tabular}{|c|c|c|c|c|c|c|c|c|c|c|c|c|}
\hline \multirow[b]{2}{*}{ Variable } & \multirow[b]{2}{*}{ Constant } & \multirow{2}{*}{$\begin{array}{c}\text { DBN } \\
\text { State } \\
\text { Yes }\end{array}$} & \multicolumn{3}{|c|}{ Age } & \multicolumn{3}{|c|}{ Income } & \multicolumn{2}{|c|}{ Education } & \multicolumn{2}{|c|}{ Race } \\
\hline & & & $45-54$ & $55-64$ & $65+$ & $\$ 25 K-\$ 49 K$ & $\$ 50 K-\$ 75 K$ & $\$ 75 K+$ & $\begin{array}{l}\text { Some } \\
\text { College }\end{array}$ & $\begin{array}{c}\text { College } \\
\text { Grad }\end{array}$ & NH Black & Hispanic \\
\hline \multicolumn{13}{|l|}{ Among all women } \\
\hline $\begin{array}{l}\text { Heard of breast density? } \\
(N=428)\end{array}$ & $\begin{array}{c}69.69 \\
{[4.31} \\
1125.66]\end{array}$ & {$\left[\begin{array}{c}1.27 \\
0.61,2.63]\end{array}\right.$} & $\left|\begin{array}{c}0.02 \\
{[0.00,0.26]}\end{array}\right|$ & $\left|\begin{array}{c}0.02 \\
{[0.00,0.27]}\end{array}\right|$ & $\begin{array}{r}0.02 \\
{[0.00} \\
0.33]\end{array}$ & $\left|\begin{array}{c}3.09 \\
{[1.27,7.52]}\end{array}\right|$ & $\mid \begin{array}{c}2.47 \\
{[0.88,6.92]}\end{array}$ & $\begin{array}{c}3.34 \\
{[1.28,8.75]}\end{array}$ & $\begin{array}{c}1.44 \\
{[0.64,3.26]}\end{array}$ & $\left|\begin{array}{c}3.15 \\
{[1.28,7.70]}\end{array}\right|$ & $\begin{array}{r}0.35 \\
{[0.15} \\
0.79]\end{array}$ & $\begin{array}{r}0.18 \\
{[0.06} \\
0.53]\end{array}$ \\
\hline \multicolumn{13}{|c|}{ Among women who have heard of breast density } \\
\hline $\begin{array}{l}\text { Does breast density mean } \\
\text { how breasts feel when you } \\
\text { touch them? (responding } \\
\text { 'yes', which is incorrect) } \\
(N=321)\end{array}$ & $\begin{array}{r}1.58 \\
{[0.39} \\
6.35]\end{array}$ & {$\left[\begin{array}{c}0.49 \\
0.26,0.91]\end{array}\right.$} & $\begin{array}{c}0.59 \\
{[0.16,2.21]}\end{array}$ & {$\left[\begin{array}{c}0.56 \\
{[0.16,1.99]}\end{array}\right.$} & $\begin{array}{c}0.82 \\
{[0.24} \\
2.84]\end{array}$ & $\mid \begin{array}{c}1.27 \\
{[0.51,3.18]}\end{array}$ & $\begin{array}{c}1.16 \\
{[0.39,3.38]}\end{array}$ & {$\left[\begin{array}{c}0.70 \\
{[0.26,1.88]}\end{array}\right.$} & {$\left[\begin{array}{c}0.53 \\
{[0.24,1.19]}\end{array}\right.$} & {$\left[\begin{array}{c}0.48 \\
{[0.22,1.04]}\end{array}\right.$} & $\begin{array}{r}2.27 \\
{[0.82} \\
6.33]\end{array}$ & $\begin{array}{r}5.33 \\
{[1.58} \\
18.01]\end{array}$ \\
\hline $\begin{array}{l}\text { Does breast density mean } \\
\text { what breasts look like on a } \\
\text { mammogram? }(N=318)\end{array}$ & $\begin{array}{r}0.65 \\
{[0.10} \\
4.12]\end{array}$ & $\begin{array}{c}1.68 \\
0.69,4.06]\end{array}$ & $\begin{array}{r}4.04 \\
{[0.73} \\
22.48]\end{array}$ & $\begin{array}{l}2.22 \\
{[0.47} \\
10.48]\end{array}$ & $\begin{array}{r}4.60 \\
{[1.03} \\
20.55]\end{array}$ & $\begin{array}{c}5.92 \\
{[1.73} \\
20.28]\end{array}$ & {$\left[\begin{array}{c}5.83 \\
{[1.41,24.06]}\end{array}\right.$} & $\begin{array}{l}3.44 \\
{[1.14} \\
10.32]\end{array}$ & $\begin{array}{c}0.87 \\
{[0.31,2.46]}\end{array}$ & {$\left[\begin{array}{c}0.91 \\
{[0.32,2.63]}\end{array}\right.$} & $\begin{array}{r}1.01 \\
{[0.18} \\
5.73]\end{array}$ & empty \\
\hline $\begin{array}{l}\text { Does breast density make it } \\
\text { more difficult for mammo to } \\
\text { correctly detect } B C ?(N=308)\end{array}$ & $\begin{array}{l}11.49 \\
{[1.54} \\
85.95]\end{array}$ & {$\left[\begin{array}{c}0.58 \\
0.22,1.48]\end{array}\right.$} & $\begin{array}{l}0.85 \\
{[0.14} \\
5.15]\end{array}$ & $\begin{array}{c}1.05 \\
{[0.16,6.72]}\end{array}$ & $\begin{array}{r}0.34 \\
{[0.06} \\
1.94]\end{array}$ & $\begin{array}{c}1.50 \\
{[0.52,4.35]}\end{array}$ & $\begin{array}{c}2.87 \\
0.63,12.98]\end{array}$ & $\begin{array}{c}2.85 \\
{[0.89,9.16]}\end{array}$ & {$\left[\begin{array}{c}0.49 \\
{[0.19,1.28]}\end{array}\right.$} & {$\left[\begin{array}{c}1.08 \\
{[0.42,2.73]}\end{array}\right.$} & $\begin{array}{r}0.35 \\
{[0.11} \\
1.10]\end{array}$ & $\begin{array}{c}0.57 \\
{[0.12,2.62]}\end{array}$ \\
\hline $\begin{array}{l}\text { Do } D B s \text { increase risk of } B C ? \\
(N=283)\end{array}$ & $\begin{array}{r}0.39 \\
{[0.08} \\
1.90] \\
\end{array}$ & $\begin{array}{c}1.34 \\
0.71,2.52]\end{array}$ & $\begin{array}{l}1.00 \\
{[1.29} \\
3.49] \\
\end{array}$ & $\mid \begin{array}{c}0.82 \\
{[0.25,2.76]}\end{array}$ & $\begin{array}{r}1.03 \\
{[0.31} \\
3.44] \\
\end{array}$ & $\begin{array}{c}1.25 \\
{[0.45,3.50]}\end{array}$ & $\begin{array}{c}1.64 \\
0.53,5.07]\end{array}$ & $\begin{array}{c}1.00 \\
{[0.36,2.75]}\end{array}$ & $\begin{array}{c}2.37 \\
{[0.99,5.66]}\end{array}$ & {$\left[\begin{array}{c}1.50 \\
{[0.65,3.47]}\end{array}\right.$} & $\begin{array}{r}1.20 \\
{[0.43} \\
3.33] \\
\end{array}$ & $\begin{array}{c}1.55 \\
{[0.36,6.61]}\end{array}$ \\
\hline \multicolumn{13}{|l|}{ Among women receiving a DBN } \\
\hline $\begin{array}{l}\text { Did you discuss breast } \\
\text { density information with a } \\
\text { doctor? }(\mathrm{N}=170)\end{array}$ & $\begin{array}{r}1.35 \\
{[0.29} \\
6.36] \\
\end{array}$ & $\mathrm{n} / \mathrm{a}$ & $\left|\begin{array}{c}0.73 \\
{[0.16,3.46]}\end{array}\right|$ & $\begin{array}{r}0.62 \\
{[0.14} \\
2.78] \\
\end{array}$ & $\begin{array}{r}0.54 \\
{[0.12} \\
2.36] \\
\end{array}$ & $\begin{array}{c}1.08 \\
{[0.35,3.36]}\end{array}$ & $\begin{array}{r}2.91 \\
{[0.79} \\
10.78] \\
\end{array}$ & $\begin{array}{r}1.08 \\
{[0.32} \\
3.58] \\
\end{array}$ & $\begin{array}{r}0.46 \\
{[0.17} \\
1.26] \\
\end{array}$ & $\begin{array}{l}0.76 \\
{[0.27} \\
2.18] \\
\end{array}$ & $\begin{array}{r}1.90 \\
{[0.77} \\
4.67] \\
\end{array}$ & $\begin{array}{l}0.86 \\
{[0.22} \\
3.31]\end{array}$ \\
\hline \multicolumn{13}{|c|}{ Among women receiving a DBN who did not yet discuss breast density info with a doctor } \\
\hline $\begin{array}{l}\text { Do you plan to discuss breast } \\
\text { density with a doctor? } \\
(\mathrm{N}=92)\end{array}$ & $\begin{array}{c}95.85 \\
{[2.74} \\
3355.03]\end{array}$ & $\mathrm{n} / \mathrm{a}$ & $\begin{array}{r}5.86 \\
{[0.48} \\
71.77]\end{array}$ & $\begin{array}{l}0.57 \\
{[0.04} \\
7.36]\end{array}$ & $\begin{array}{r}2.71 \\
{[0.25} \\
29.21]\end{array}$ & $\begin{array}{r}0.01 \\
{[0.00} \\
0.22]\end{array}$ & $\left|\begin{array}{c}0.01 \\
{[0.00,0.14]}\end{array}\right|$ & $\begin{array}{r}0.00 \\
{[0.00} \\
0.07]\end{array}$ & $\begin{array}{r}0.25 \\
{[0.30} \\
2.02]\end{array}$ & $\left.\mid \begin{array}{c}0.39 \\
{[0.05,2.95]}\end{array}\right]$ & $\begin{array}{r}8.53 \\
{[0.98} \\
74.10]\end{array}$ & $\begin{array}{c}13.26 \\
{[1.25} \\
140.36]\end{array}$ \\
\hline \multicolumn{13}{|c|}{ Among women receiving a DBN AND who heard of breast density, does knowing about breast density make you feel } \\
\hline $\begin{array}{l}\text {-very/somewhat anxious } \\
(\mathrm{N}=135)\end{array}$ & $\begin{array}{r}0.34 \\
{[0.05} \\
2.27]\end{array}$ & $\mathrm{n} / \mathrm{a}$ & $\left|\begin{array}{c}0.47 \\
{[0.05,4.34]}\end{array}\right|$ & $\left|\begin{array}{c}0.21 \\
{[0.33,1.33]}\end{array}\right|$ & $\begin{array}{c}1.06 \\
{[0.20} \\
5.60] \\
\end{array}$ & $\left|\begin{array}{c}0.36 \\
{[0.10,1.30]}\end{array}\right|$ & $\left.\begin{array}{c}0.77 \\
{[0.11,5.38]}\end{array}\right]$ & $\begin{array}{c}0.57 \\
{[0.10,} \\
3.35]\end{array}$ & {$\left[\begin{array}{c}1.98 \\
{[0.54,7.20]}\end{array}\right.$} & $\begin{array}{c}0.71 \\
{[0.17,3.05]}\end{array}$ & $\begin{array}{l}15.28 \\
{[4.22} \\
55.27]\end{array}$ & $\begin{array}{c}1.83 \\
{[0.25} \\
13.23] \\
\end{array}$ \\
\hline $\begin{array}{l}\text { - very/somewhat informed } \\
(\mathrm{N}=130)\end{array}$ & $\begin{array}{r}4.90 \\
{[0.94,} \\
25.42]\end{array}$ & $\mathrm{n} / \mathrm{a}$ & $\begin{array}{c}1.77 \\
{[0.31} \\
10.21]\end{array}$ & \begin{tabular}{|c|}
3.61 \\
{$[0.66,19.76$} \\
]
\end{tabular} & $\begin{array}{l}1.03 \\
{[0.22} \\
4.69]\end{array}$ & $\mid \begin{array}{c}0.58 \\
{[0.06,5.47]}\end{array}$ & $\mid \begin{array}{c}0.47 \\
{[0.05,4.07]}\end{array}$ & $\begin{array}{c}0.20 \\
{[0.03,1.53]}\end{array}$ & $\begin{array}{c}1.27 \\
{[0.21,7.75]}\end{array}$ & $\mid \begin{array}{c}1.55 \\
{[0.31,7.64]}\end{array}$ & $\begin{array}{c}3.46 \\
{[0.44,} \\
27.33]\end{array}$ & $\begin{array}{c}0.74 \\
{[0.07,8.31]}\end{array}$ \\
\hline $\begin{array}{l}\text { - very/somewhat confused } \\
(\mathrm{N}=110)\end{array}$ & $\begin{array}{c}0.14 \\
{[0.02} \\
1.02]\end{array}$ & $\mathrm{n} / \mathrm{a}$ & $\begin{array}{r}1.70 \\
{[0.20} \\
14.55]\end{array}$ & $\begin{array}{c}0.94 \\
{[0.16,} \\
5.69]\end{array}$ & empty & $\left|\begin{array}{c}0.06 \\
{[0.00,0.77]}\end{array}\right|$ & $\begin{array}{c}0.28 \\
{[0.03,2.38]}\end{array}$ & $\begin{array}{c}0.03 \\
{[0.00,0.40]}\end{array}$ & $\begin{array}{r}2.59 \\
{[0.34} \\
19.85]\end{array}$ & $\begin{array}{c}3.71 \\
{[0.41} \\
33.94]\end{array}$ & $\begin{array}{c}6.66[1.31 \\
33.92]\end{array}$ & empty \\
\hline
\end{tabular}

Boxes filled with red indicate significant undesirable outcomes; boxes filled with green indicate significant desirable outcomes.

Boxes filled with red indicate significant undesirable outcomes; boxes filled with green indicate significant desirable outcomes

Constant estimates baseline odds for reference groups (non-DBN state, $<45,<\$ 25 K$, no college, White)

Odds ratios shown with $95 \%$ confidence intervals in brackets

Significant effects at the $p<0.05$ level are in bold

'empty' signifies conditions excluded from the model due to having no responses or non-varying responses

All dichotomous outcome variables coded as $1=$ yes, $0=$ no

Our results showing no state DBN status differences in discussing $\mathrm{BD}$ with a provider differ from those from a recent online survey ${ }^{5}$ regarding discussions between women with dense breasts and their provider about having additional screening, where women in states with notification laws were more likely to have had such discussions. However, those online survey respondents had higher education and incomes. Our finding of few other differences by state legislation status are similar to other national surveys. ${ }^{2}$

Our study had a relatively small sample size, reducing statistical power and wherewithal to examine effects of specific states' DBN wording, but the differences in outcomes we observed for vulnerable women suggest that future DBNs should address these gaps to optimize outcomes. Given the discordance of state DBNs' literacy levels and readability with 
their populations' levels, ${ }^{6}$ the present findings underscore the importance of crafting federal DBNs in clear, plain language, and rigorously testing the proposed language with diverse samples, to ensure that the positive goals of increased BD awareness, knowledge, and discussions with physicians about personal risk for breast cancer, and supplemental screening appropriate to such risk, are achieved for all women.

Corresponding Author: Nancy R. Kressin, PhD; Section of General Internal Medicine, Department of Medicine Boston University School of Medicine, Boston, MA, USA (e-mail: nkressin@bu.edu).

Funding Information This work was supported by a pilot grant from the Boston University Clinical and Translational Science Institute (1UL1TR001430). Dr. Kressin is supported in part by a Senior VA Health Services Research Career Scientist award from the Department of Veterans Affairs, Health Services Research \& Development Service (RCS 02-066-1). Dr. Gunn receives support from the National Cancer Institute (1K07CA221899-01A1).

\section{Compliance with Ethical Standards:}

Conflict of Interest: The authors declare that they do not have a conflict of interest.

\section{REFERENCES}

1. Food and Drug Administration, H. Mammography Quality Standards Act. 2019; Available from: https://www.federalregister.gov/documents/2019/ 03/28/2019-05803/mammography-quality-standards-act.

2. Rhodes DJ, et al. Awareness of breast density and its impact on breast cancer detection and risk. J Clin Oncol. 2015;33(10):1143-1150.

3. Manning $\mathbf{M}$, et al. Influences of race and breast density on related cognitive and emotion outcomes before mandated breast density notification. Soc Sci Med. 2016;169:171-179.

4. Gunn C, et al. Women's Perceptions of Dense Breast Notifications: "So what is that supposed to mean?". Patient Educ Couns. 2018;101(6):11231129.

5. Cappello NM, Richetelli D, Lee CI. The Impact of Breast Density Reporting Laws on Women's Awareness of Density-Associated Risks and Conversations Regarding Supplemental Screening With Providers. J Am Coll Radiol. 2019;16(2): 139-146.

6. Kressin NR, Gunn CM, Battaglia TA. Content, Readability, and Understandability of Dense Breast Notifications by State. Jama. 2016;315(16):1786-1788.

Publisher's Note Springer Nature remains neutral with regard to jurisdictional claims in published maps and institutional affiliations. 